\title{
How do community hospitals perform in acute ischemic stroke treatment in the rt-PA era?
}

\author{
NisaVorasoot, ${ }^{1,2}$ Kannikar Kongbunkiat, ${ }^{1,2}$ Udomluck Pansukwech, ${ }^{1}$ Kittisak Sawanyawisuth, $1,3,4$ \\ SomsakTiamkao, 1,2 Narongrit Kasemsap 1,2 \\ ${ }^{1}$ Department of Medicine, Faculty of Medicine, Khon Kaen University, Khon Kaen \\ 2 North-eastern Stroke Research Group \\ ${ }^{3}$ Research Center in Back, Neck, Other Joint Pain and Human Performance (BNOJPH) \\ ${ }^{4}$ Internal Medicine Research Group
}

Background : The Stroke Fast Track Network, which has been development in Thailand to improve stroke care, particularly in community hospitals $(\mathrm{CH})$. This study, aimed to evaluate the quality of acute ischemic stroke care in the various hospital levels.

Methods : Data used in this study were randomly selected from the medical records, which are sent to the National Health Security Office for reimbursement purposes between October 2015 and August 2016. Patient demographics, risk factors, stroke subtypes, stroke severity, quality of care indicators and complications were recorded. Pair comparisons between two groups were carried out using Bonferroni correction.

Table 1 Demographic and baseline characteristics

\begin{tabular}{|c|c|c|c|c|c|}
\hline \multirow[b]{2}{*}{ Characteristics } & \multicolumn{4}{|c|}{ Hospital levels } & \multirow[t]{2}{*}{ p-value } \\
\hline & $\begin{array}{c}\text { CHs } \\
(n=169)\end{array}$ & $\begin{array}{c}\text { RHs } \\
(\mathrm{n}=629)\end{array}$ & $\begin{array}{c}\text { THs } \\
(n=149)\end{array}$ & $\begin{array}{c}\text { Total } \\
(\mathrm{n}=947)\end{array}$ & \\
\hline Male, n (\%) & $95(56.2)$ & $311(49.4)$ & $71(47.7)$ & $477(50.4)$ & 0.23 \\
\hline $\begin{array}{l}\text { Age (years), median (IQR) } \\
\text { Risk factors, } \mathbf{n}(\%)\end{array}$ & $70(61-76)$ & $66(56-75)$ & $67(58-74)$ & $67(58-75)$ & $<0.01^{a, b}$ \\
\hline Diabetes mellitus & $49(29.0)$ & $161(25.6)$ & $41(27.5)$ & $251(26.5)$ & 0.64 \\
\hline Hypertension & $82(48.5)$ & $307(48.8)$ & $74(49.7)$ & $463(48.9)$ & 0.97 \\
\hline Dyslipidemia & $56(33.1)$ & $138(21.9)$ & $47(31.5)$ & $241(25.4)$ & $<0.01^{\mathrm{a}}$ \\
\hline Chronic kidney disease & $2(1.2)$ & $32(5.1)$ & $8(5.4)$ & $42(4.4)$ & 0.08 \\
\hline Atrial fibrillation & $39(23.1)$ & $144(22.9)$ & $44(29.5)$ & $227(24.0)$ & 0.38 \\
\hline Coronary artery disease & $5(3.0)$ & $39(6.2)$ & $8(5.4)$ & $52(5.5)$ & 0.26 \\
\hline Previous TIA/stroke & $22(13.0)$ & $84(13.4)$ & $17(11.4)$ & $123(13.0)$ & 0.82 \\
\hline $\begin{array}{l}\text { Smoking } \\
\text { Stroke subtype, } \mathbf{n}(\%)\end{array}$ & $12(7.1)$ & $82(13)$ & $21(14.1)$ & $115(12.1)$ & 0.08 \\
\hline LAA & $52(30.8)$ & $235(37.4)$ & $45(30.2)$ & $332(35.1)$ & 0.11 \\
\hline $\mathrm{CE}$ & $42(24.9)$ & $164(26.1)$ & $50(33.6)$ & $256(27.0)$ & 0.14 \\
\hline SVD & $74(43.8)$ & $224(35.6)$ & $47(31.5)$ & $345(36.4)$ & 0.06 \\
\hline Other determined & $0(0.0)$ & $1(0.2)$ & $1(0.7)$ & $2(0.2)$ & 0.32 \\
\hline Undetermined & $1(0.6)$ & $3(0.5)$ & $4(2.7)$ & $8(0.8)$ & $0.04 \mathrm{c}$ \\
\hline $\begin{array}{l}\text { Initial NIHSS, median } \\
\text { (IQR) }\end{array}$ & $6(3-10)$ & $8(5-13)$ & $9(6-15)$ & $8(4-14)$ & $<0.01^{\mathrm{a}, \mathrm{b}}$ \\
\hline EKG monitoring, n (\%) & $74(43.8)$ & $257(40.9)$ & $72(48.3)$ & $403(42.6)$ & 0.24 \\
\hline Vascular study, n (\%) & $1(0.7)$ & $17(2.9)$ & $27(18.4)$ & $45(5.0)$ & $<0.01^{b, c}$ \\
\hline
\end{tabular}

$a$ :CHs vs. RHs ( $p<0.05), b$ :CHs vs. THs $(p<0.05)$,

c: RHs vs. THs $(p<0.05)$

Narongrit Kasemsap, M.D., Department of Medicine, Faculty of Medicine, Khon Kaen University, Khon Kaen, 40002, Thailand, Tel. 66-43-363664 Fax. 66-43-347542 Email: naroka@kku.ac.th
Results : A total of 947 patients, 169 patients from CHs, 629 from regional hospitals $(\mathrm{RH})$ and 149 from tertiary hospital (TH) were included in the final analysis. The $\mathrm{CH}$ group had a higher median age but lower median initial NIHSS than the RH and TH groups (median age $=70,66$ and 67 years, respectively, and initial NIHSS $=6,8$, and 9, respectively). The $\mathrm{CH}$ group had shorter onset-toneedle times for rt-PA than the other two groups (147 vs 178.5 and $180 \mathrm{~min}$ ). The $\mathrm{CH}$ group had a lower proportion of asymptomatic intracerebral hemorrhage $(0.6 \%)$, and lower mortality rate $(1.2 \%)$ than either the $\mathrm{RH}$ or TH group.

Conclusion : Community hospitals have the potential care for acute ischemic stroke patients in the same way as $\mathrm{RH}$ or THs.

Table 2 Quality measurement and stroke outcome

\begin{tabular}{|c|c|c|c|c|c|}
\hline \multirow{2}{*}{ Acute stroke care indicators } & \multicolumn{4}{|c|}{ Hospital levels } & \multirow[t]{2}{*}{ p-value } \\
\hline & CHs (n=169) & RHs $(n=629)$ & THs $(n=149)$ & Total $(n=947)$ & \\
\hline $\begin{array}{l}\text { Onset to needle (min), } \\
\text { median (IQR) }\end{array}$ & $147(120-194)$ & $\begin{array}{c}178.5 \\
(135-210)\end{array}$ & $180(143-225)$ & $174.5(135-210)$ & $0.01^{\mathrm{a}, \mathrm{b}}$ \\
\hline $\begin{array}{l}\text { Door to needle (min), median } \\
\text { (IQR) }\end{array}$ & $55(40-69)$ & $60(45-80)$ & $73(55-94)$ & $60(48-82)$ & $<0.01 \mathrm{~b}, \mathrm{c}$ \\
\hline $\begin{array}{l}\text { Antiplatelet within } 48 \text { hours, } \\
\text { n (\%) }\end{array}$ & $142(84.0)$ & 463 (73.6) & $94(63.1)$ & $699(73.8)$ & $<0.01^{\mathrm{a}, \mathrm{b}, \mathrm{c}}$ \\
\hline Rehabilitations, n (\%) & $125(74.0)$ & $490(77.9)$ & $113(75.8)$ & $728(76.9)$ & 0.53 \\
\hline Complications, n (\%) & & & & & \\
\hline Pneumonia & $30(17.8)$ & $155(24.6)$ & $38(25.5)$ & $223(23.5)$ & 0.14 \\
\hline Urinary tract infection & $4(2.4)$ & $38(6.0)$ & $6(4.0)$ & $48(5.1)$ & 0.14 \\
\hline Sepsis & $7(4.1)$ & $34(5.4)$ & $11(7.4)$ & $52(5.5)$ & 0.44 \\
\hline Gastrointestinal bleeding & $3(1.8)$ & $21(3.3)$ & $1(0.7)$ & $25(2.6)$ & 0.18 \\
\hline Acute kidney injury & $5(3.0)$ & $27(4.3)$ & $5(3.4)$ & $37(3.9)$ & 0.80 \\
\hline Myocardial infarction & $0(0.0)$ & $1(0.2)$ & $1(0.7)$ & $2(0.2)$ & 0.32 \\
\hline Heart failure & $6(3.6)$ & $14(2.2)$ & $2(1.3)$ & $22(2.3)$ & 0.44 \\
\hline Brain herniation & $4(2.4)$ & $33(5.2)$ & $12(8.1)$ & $49(5.2)$ & 0.07 \\
\hline Asymptomatic ICH & $1(0.6)$ & $31(4.9)$ & $5(3.4)$ & $37(3.9)$ & $0.01^{\mathrm{a}}$ \\
\hline Symptomatic ICH & $5(3.0)$ & $27(4.3)$ & $4(2.7)$ & $36(3.8)$ & 0.62 \\
\hline Cost (baht), median (IQR) & $\begin{array}{c}24,400 \\
(12,654-50,376)\end{array}$ & $\begin{array}{c}39,506 \\
(17,051-64,315)\end{array}$ & $\begin{array}{c}53,649 \\
(28,798-79,320)\end{array}$ & $\begin{array}{c}39,656 \\
(16,689-64,805)\end{array}$ & $<0.01^{\mathrm{a}, \mathrm{b}, \mathrm{c}}$ \\
\hline $\begin{array}{l}\text { Length of hospital stay (day), } \\
\text { median (IQR) }\end{array}$ & $5(4-6)$ & $5(4-7)$ & $5(4-8)$ & $5(4-7)$ & 0.19 \\
\hline Discharge status, n (\%) & & & & & $<0.01^{b, c}$ \\
\hline Complete recovery & $1(0.6)$ & $2(0.3)$ & $4(2.7)$ & $7(0.7)$ & \\
\hline Improved & $145(85.8)$ & $515(81.9)$ & $118(79.2)$ & $778(82.2)$ & \\
\hline Not improved & $21(12.4)$ & $80(12.7)$ & $12(8.1)$ & $113(11.9)$ & \\
\hline Death & $2(1.2)$ & $32(5.1)$ & $15(10.1)$ & $49(5.2)$ & \\
\hline
\end{tabular}

